\title{
Implementing a multilevel intervention to accelerate colorectal cancer screening and follow-up in federally qualified health centers using a stepped wedge design: a study protocol
}

\author{
Karen Kim', Blasé Polite², Donald Hedeker ${ }^{3}$, David Liebovitz ${ }^{4}$, Fornessa Randal', Manasi Jayaprakash',
} Michael Quinn ${ }^{5}$, Sang Mee Lee ${ }^{3}$ and Helen Lam ${ }^{1 *}$ (D)

\begin{abstract}
Background: Screening for colorectal cancer (CRC) not only detects disease early when treatment is more effective but also prevents cancer by finding and removing precancerous polyps. Because many of our nation's most disadvantaged and vulnerable individuals obtain health care at federally qualified health centers, these centers play a significant role in increasing CRC screening among the most vulnerable populations. Furthermore, the full benefits of cancer screenings must include timely and appropriate follow-up of abnormal results. Thus, the purpose of this study is to implement a multilevel intervention to increase rates of CRC screening, follow-up, and referral-to-care in federally qualified health centers, as well as simultaneously to observe and to gather information on the implementation process to improve the adoption, implementation, and sustainment of the intervention. The multilevel intervention will target three different levels of influences: organization, provider, and individual. It will have multiple components, including provider and staff education, provider reminder, provider assessment and feedback, patient reminder, and patient navigation.

Methods: This study is a multilevel, three-phase, stepped wedge cluster randomized trial with four clusters of clinics from four different FQHC systems. In the first phase, there will be a 3-month waiting period during which no intervention components will be implemented. After the 3-month waiting period, we will randomize two clusters to cross from the control to the intervention and the remaining two clusters to follow 3 months later. All clusters will stay at the same phase for 9 months, followed by a 3-month transition period, and then cross over to the next phase.

* Correspondence: hvallina@medicine.bsd.uchicago.edu

'Center for Asian Health Equity, University of Chicago, 5841 S. Maryland Ave, MC1140, Chicago, IL 60637, USA

Full list of author information is available at the end of the article

(c) The Author(s). 2020 Open Access This article is licensed under a Creative Commons Attribution 4.0 International License, which permits use, sharing, adaptation, distribution and reproduction in any medium or format, as long as you give appropriate credit to the original author(s) and the source, provide a link to the Creative Commons licence, and indicate if changes were made. The images or other third party material in this article are included in the article's Creative Commons licence, unless indicated otherwise in a credit line to the material. If material is not included in the article's Creative Commons licence and your intended use is not permitted by statutory regulation or exceeds the permitted use, you will need to obtain permission directly from the copyright holder. To view a copy of this licence, visit http://creativecommons.org/licenses/by/4.0/. The Creative Commons Public Domain Dedication waiver (http://creativecommons.org/publicdomain/zero/1.0/) applies to the data made available in this article, unless otherwise stated in a credit line to the data. 
(Continued from previous page)

Discussion: There is a pressing need to reduce disparities in CRC outcomes, especially among racial/ethnic minority populations and among populations who live in poverty. Single-level interventions are often insufficient to lead to sustainable changes. Multilevel interventions, which target two or more levels of changes, are needed to address multilevel contextual influences simultaneously. Multilevel interventions with multiple components will affect not only the desired outcomes but also each other. How to take advantage of multilevel interventions and how to implement such interventions and evaluate their effectiveness are the ultimate goals of this study.

Trial registration: This protocol is registered at clinicaltrials.gov (NCT04514341) on 14 August 2020.

Keywords: Colorectal cancer, Multilevel intervention, Stepped wedge design, Implementation strategy, Federally qualified health center, $\mathrm{FQHC}$

\section{Contributions to the literature}

- Study findings will provide the evidence base for multilevel interventions to increase rates of colorectal cancer screening and follow-up care among racial/ethnic minority and lowincome populations, which disproportionately receive health care in federally qualified health centers.

- Although the parallel cluster-randomized trial is a gold standard for causal inference, it may not be feasible in real-world settings. The stepped wedge design retains some elements of randomization, allows multiple data collection points, and enhances the precision of the study. This study will provide evidence of the flexibility and feasibility of a stepped wedge design in multilevel interventional studies.

- The effectiveness of an intervention, in part, depends on the strength of the implementation process. This study will demonstrate how to use the Consolidated Framework for Implementation Research (CFIR) to conduct an ongoing evaluation of the implementation process.

\section{Background}

\section{Colorectal cancer control and safety-net health systems}

Colorectal cancer is the second leading cause of cancer death in the United States [1]. Screening for CRC not only detects disease early when treatment is more effective but also prevents cancer by finding and removing precancerous polyps. Although CRC screening is effective in preventing and reducing CRC mortality, it remains underutilized. Despite strong evidence to support CRC screening, nationally, only $68.8 \%$ of adults had up-todate screening in 2018 [2]. While the percentage of ageeligible adults who are up-to-date with recommended CRC screening has been increasing over the years, nearly $28 \%$ of the eligible adults have never been screened, and this figure is even higher among racial/ethnic minorities and people who live in poverty [3-6].
Individuals who are without health insurance or a regular care provider are more likely to have never been screened than those with health insurance and a regular care provider [3]. Racial/ethnic minorities and people with low social-economic status (SES) are often among those who lack health insurance and a regular source of care. Therefore, the underuse of CRC screening is more frequent among these populations [4-6]. These populations also disproportionately receive health care in safety-net settings, such as federally qualified health centers (FQHCs) [7, 8]. FQHCs are designed to provide comprehensive, quality primary healthcare services to medically underserved communities and vulnerable populations. In 2018, FQHCs served 28 million patients, of whom $23 \%$ were uninsured, $63 \%$ were racial/ethnic minorities, and $91 \%$ were living below $200 \%$ poverty level [9]. Because many of our nation's most disadvantaged and vulnerable individuals obtain health care at FQHCs, these health centers play a significant role in increasing CRC screening among the most vulnerable populations.

\section{Challenges of colorectal cancer control in safety-net healthcare systems}

Due to the cost and limited availability of specialty services combined with patient preferences, safety-net settings often promote non-invasive screening methods, such as fecal occult blood test (FOBT) or fecal immunochemical tests (FIT), as the modality for screening [1013]. To achieve the benefits of CRC screening using FOBT/FIT, timely follow-up of positive results must occur. Failure to offer or complete a diagnostic evaluation following a positive FOBT/FIT has several consequences, including late-stage CRC diagnoses. This failure could undermine the benefits of screening and increase disparities [14-17]. While no national estimates of the proportion of individuals without follow-up diagnostic evaluation after receiving a positive FOBT/FIT exist, several studies report follow-up rates ranging from less than 50 to $90 \%$ within 1 year of a positive test [1827]. Furthermore, follow-up rates varied across 
healthcare systems, with integrated healthcare systems exhibiting higher follow-up rates by 12 months (82-86\%) compared with safety-net health systems (56-58\%) [22, 28-30]. Safety-net healthcare systems not only face challenges to increase CRC screening rates among racial/ethnic minorities and low SES patients but also to increase follow-up diagnostic evaluation rates after a positive FOBT/FIT.

\section{Multilevel influences on cancer screening behavior}

Individuals live and seek care in a complex environment, and multiple levels of contextual influences may affect individual decisions and actions [31, 32]. Traditionally, behavior change models, such as the Theory of Planned Behavior Model (TPB), mainly focus on the individual and do not explicitly show how contextual factors may affect and interact with the individual. By incorporating system models, such as the Social Ecological Model, into an individual behavior change model, we can then expand such models to consider multilevel contextual influences, which affect the individual through interdependent interaction [33]. Figure 1 shows the integrated Social Ecological Model and the TPB Model, which illustrates a more holistic review of cancer screening behavior. Multilevel interventions target changes in more than one contextual level (e.g., organization, provider, and patient levels) to influence health behavior, healthcare practice, and health outcomes [34-36]. Although the call for multilevel interventions has increased [36-39], there is still a lack of evidence addressing how to implement multilevel interventions or how interventions at multiple levels interact and affect health outcomes.

\section{Aims and objectives}

ACCSIS-Chicago (Accelerating Colorectal Cancer Screening and Follow-up Through Implementation Science - Chicago) is a 4-year study. The overall objective of this study is to provide the evidence base for multilevel interventions that increase rates of CRC screening, follow-up, and referral-to-care at federally qualified health centers, and to understand how to improve the adoption, implementation, and sustainment of these interventions. This study is built on the lessons learned from the 1-year pilot feasibility study and our 5-year CDC funded Colorectal Cancer Control and Prevention Screening Program that used a multilevel and organized approach to increase CRC screening rates among integrated healthcare systems and FQHCs. Although we obtained programmatic success in increasing CRC screening rates with our CDC funded project among our partner health systems, the process to achieve these positive outcomes and how multilevel components interact and influence each other remained unknown. In this study, we will test the effectiveness of our multilevel intervention while simultaneously observing and gathering information on the implementation process. Furthermore, we will examine how intervention components at different levels interact and influence health outcomes. The selection of our multilevel intervention components is based on extensive literature review, the strength of evidence, and findings from our previous studies and projects. The multilevel intervention will target three different levels of influence (organization, provider, and individual) to improve rates of CRC screening, follow-up, and referral-to-care at our partner FQHCs. Our multilevel intervention will have multiple components, including provider and staff education, provider reminder,

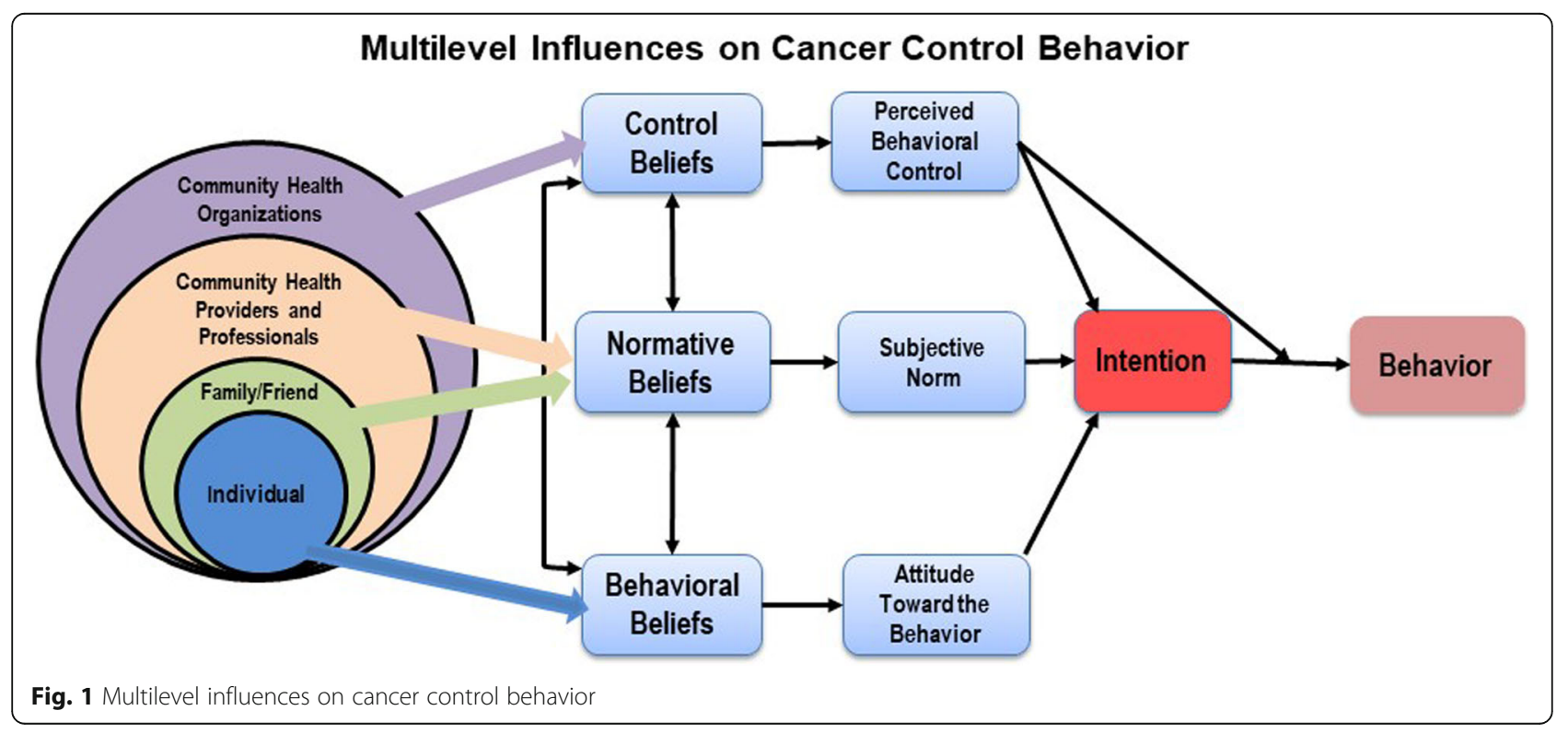


provider assessment and feedback, patient reminder, and patient navigation.

The aims of ACCSIS-Chicago are fourfold: (1) use a stepped wedge design to implement a multilevel intervention in three phases, (2) collect quarterly data to track changes over time, (3) evaluate the implementation process and the effectiveness of implementation strategies through observations, interviews, and annual survey, and (4) evaluate the effectiveness of the multilevel intervention using multilevel and longitudinal modeling.

\section{Methods}

This study is conducted as part of the NCI-funded consortium, The Accelerating CRC Screening and Followup through Implementation (ACCSIS) Program. The overall aim of ACCSIS is to conduct multi-site, coordinated, transdisciplinary research to evaluate and improve CRC screening processes using implementation science strategies. The ACCSIS-Chicago will implement a multilevel, multicomponent intervention to increase rates of CRC screening, follow-up, and referral-to-care at four federally qualified health systems located in Illinois and Indiana. The protocol has been reviewed and approved by the University of Chicago Institutional Review Board (IRB19-1496) and is registered at clinicaltrials.gov (NCT04514341).

\section{Conceptual framework}

CRC screening and follow-up processes are complex and include several steps and interfaces. However, very few interventional studies have simultaneously targeted patient-, provider-, and organization-level factors. Interventions that focus on reducing barriers across several levels will likely be more effective for increasing rates of CRC screening, follow-up, and referral-to-care. Figure 2 shows the conceptual framework for ACCSIS-Chicago and how the multilevel components work together along the CRC control and prevention continuum to achieve the desired outcomes.

\section{Multilevel intervention and implementation strategy Provider level component}

Provider education Numerous studies indicate the importance of physician recommendation in influencing a patient's CRC screening decision [40-53], and this factor is a primary predictor for patient adherence with screening guidelines [54-56]. Provider challenges for CRC screening include (1) asymptomatic patients believe that screening is unnecessary, especially when offered an invasive procedure, and (2) the different screening methods may cause confusion, which underscores the scientific ambiguity surrounding the decision [49]. Studies have shown that shared decision-making can improve patient compliance when decisions are complex, such as CRC screening [57-59], yet many clinicians lack training in shared decision-making [60]. They are often uncertain about which decisions require patient participation, and about how to communicate technical concepts to patients in simple language that is accurate, balanced, and understandable. Thus, provider education is an important component for improving CRC screening compliance. The research team will provide tailored education and guidance on best practices for participating health professionals. The educational sessions will focus on $\mathrm{CRC}$ risk assessment, screening guidelines, cultural competency, shared decision-making, and communication skills using an academic detailing approach. The academic detailing approach involves trained experts visiting healthcare professionals in their settings to provide tailored education and guidance on best practices, which have shown to have a significant effect on increasing rates of CRC screening [61-66]. Each session will last about 15 to $20 \mathrm{~min}$ and will take place during routine

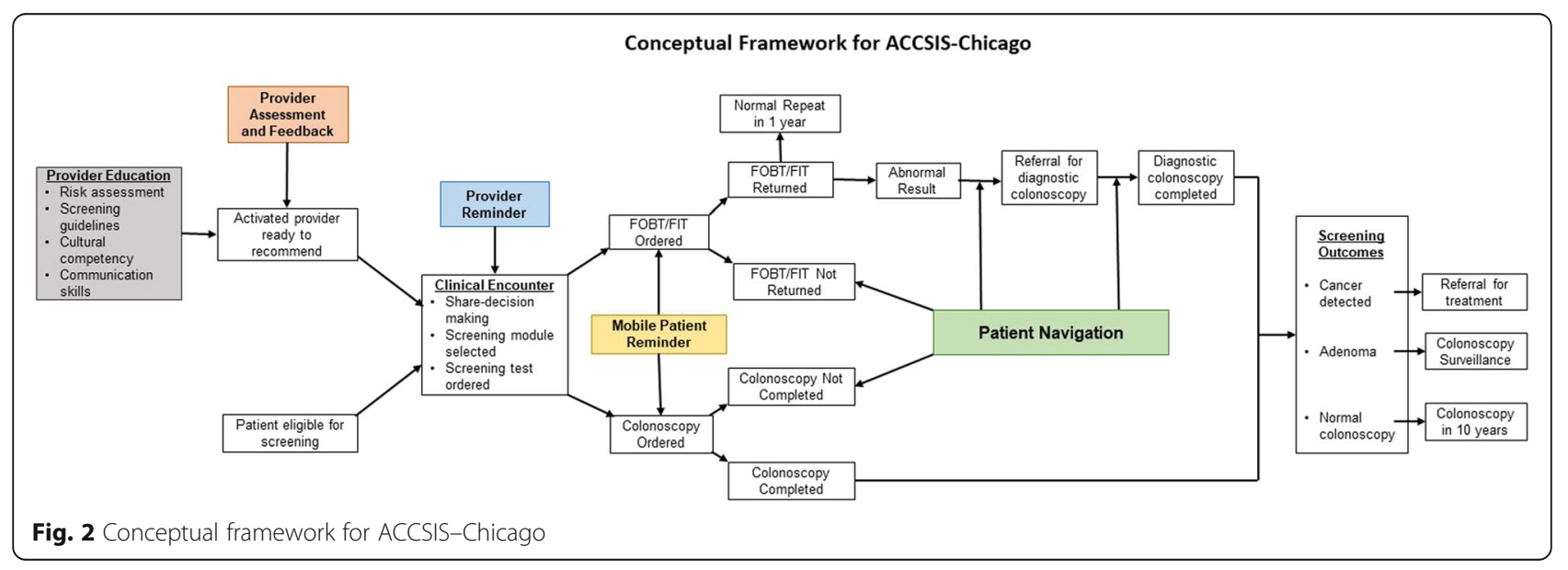


staff and provider meetings. These academic detailing sessions will be ongoing throughout the study period.

Provider assessment and feedback We will combine academic detailing with practice facilitation, which includes assessment and feedback on aggregate and individual provider screening behavior and practice performance [67]. We will capture data from the electronic health record (EHR) across our partner sites to generate clinic- and provider-specific reports. The reports will include CRC screening order rates, screening completion rates, follow-up rates for abnormal results, and referral to oncology care. Providers will be able to see each other's performances, which allows providers to compare and learn from each other's successes. Our research team will deliver the feedback reports quarterly.

Provider reminder As previously mentioned, studies have shown that provider recommendations have the most significant impact on CRC screening rates. However, given the multitude of competing priorities during a patient encounter, CRC screening recommendations can be overlooked. A provider prompt generated electronically or manually by staff members will be implemented to remind providers to screen their eligible patients. Provider prompts have been shown to increase CRC screening rates [68].

\section{Organization and individual level component}

Patient reminder Provider recommendation alone does not guarantee the completion of CRC screening, which involves patient compliance. We will implement a patient reminder component using a text-based platform, also known as short message service (SMS), to engage and remind patients to complete the screening. Patients who use a stool-based screening method will receive an initial text message with links to videos and educational materials within 3 to 5 days after the ordered test. Patients will continue to receive text messages weekly for 90 days as a reminder to complete and return the stool card for testing. When patients respond that they have returned the stool card, or when they opt out of the reminder system, the SMS will stop. For patients who use colonoscopy as a screening method, the SMS will also include instruction on bowel preparation and dietary restrictions 5 days before the procedure. Studies have demonstrated that SMS patient reminders not only had a positive impact on screening rates but also improved the quality of bowel preparation [69-74].

Patient navigation Patient navigation services will address both organization and individual influences. Patient navigation focuses on eliminating barriers by guiding a patient through a complex healthcare system, addressing education, sociocultural, and logistical needs using trained staff. Patient navigation has consistently shown an association with increased CRC screening completion [75-82] and diagnostic follow-up after a positive test result [83-87]. We will hire and train two full-time CRC navigators and adapt and modify the wellstudied New Hampshire Colorectal Cancer Screening Program (NHCRCSP) Patient Navigator Model [88-90]. Navigator training will include didactic and clinical sessions with ongoing training every quarter throughout the duration of the program. CRC Navigators will have field training at each healthcare system to become familiar with the clinic workflow and referral process, as well as an opportunity for clinical shadowing in the CRC oncology clinic and colonoscopy suite. CRC navigators will also undergo pre and post-training evaluation, and they will be centralized and supervised by the project director. All CRC navigators will meet biweekly to discuss their cases, share information, and address any issues associated with their roles and responsibilities.

\section{Implementation strategy}

The effectiveness of an intervention, in part, depends on the strength of the implementation process. A successful implementation process requires attention to critical contextual factors. From the very outset, we will conduct a pre-implementation organizational readiness assessment (ORA). We will use the CFIR framework (Consolidated Framework for Implementation Research) to guide our ORA. CFIR is a conceptual framework that was developed to conduct a systematic assessment of multilevel implementation contexts to identify factors that might influence intervention implementation and effectiveness [91]. The CFIR framework consists of five domains (intervention characteristics, inner setting, outer setting, characteristics of individuals, and processes) and a standard set of constructs that allows a comprehensive assessment. We will conduct key informant interviews with health system leadership and clinic leaders to evaluate: (1) current CRC screening workflow, (2) CRC data capturing and validation process, and (3) capacity and resources available to support the implementation. Table 1 summarizes the interview questions based on CFIR domains. Also, we will conduct a readiness survey with clinical providers and support staff to assess organizational climate and culture. Our 29 survey items were adapted and modified from validated instruments that measured CFIR constructs using a Delphi method [92-95].

Effective strategies to support the implementation process is critical. Implementation strategies are actions taken to enhance the adoption, implementation, and sustainability of evidence-based interventions. Table 2 
Table 1 Pre-implementation readiness assessment guided by CFIR domains

\section{Pre-implementation Readiness Assessment Item}

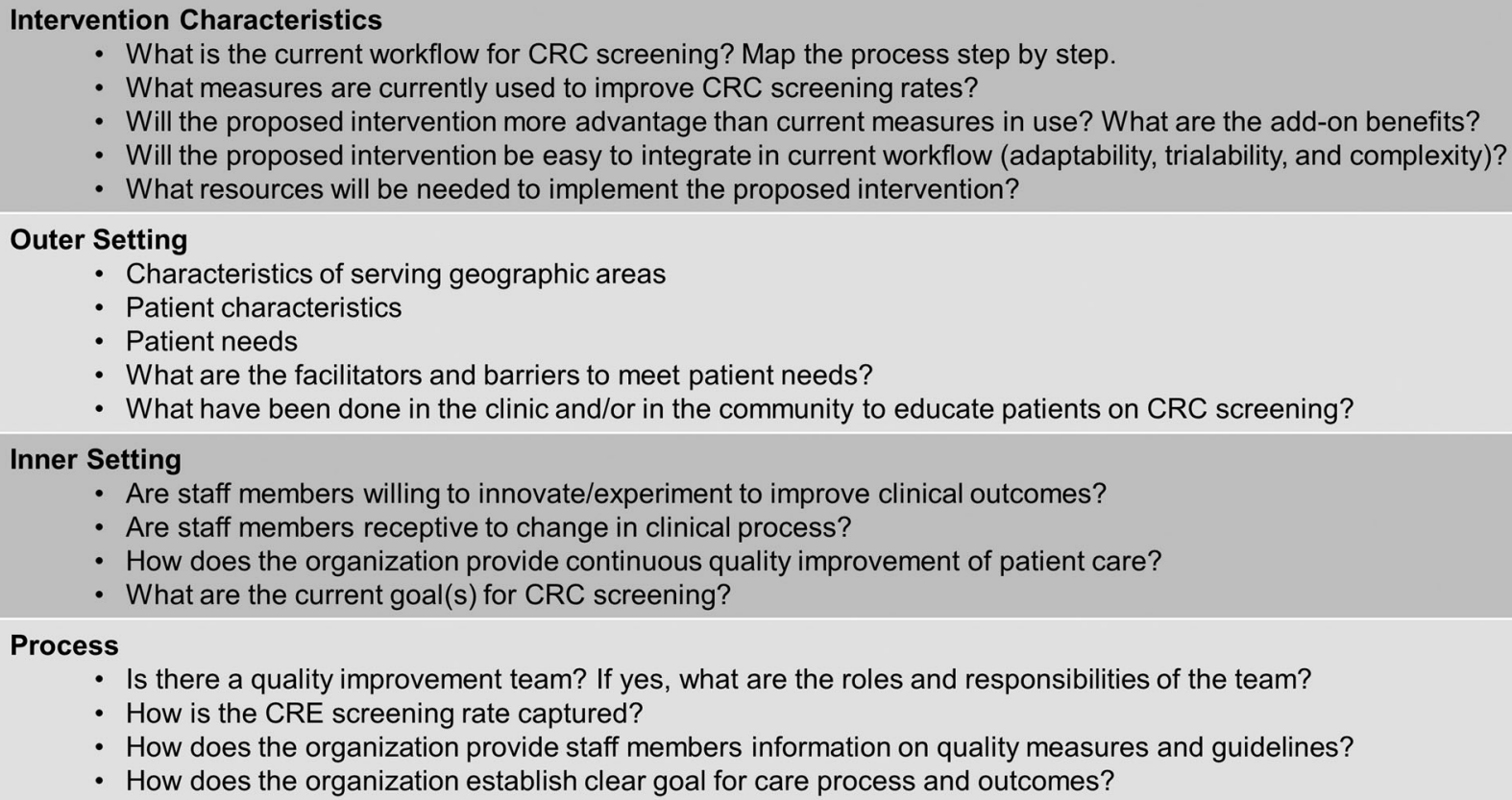

summarizes the implementation strategies that we will use to implement our multilevel intervention.

Finally, we will implement our multilevel, multicomponent intervention in three phases (Fig. 3) along the CRC screening continuum.

\section{Study design}

In the evaluation of a multilevel intervention, an important design decision is whether to assess only the combined impact of the multiple components compared to no intervention or the separate effects of each component at different levels and the possible interaction effects [96]. While a parallel cluster randomized trial is a gold standard for causal inference, it requires a large number of clusters to assess separate effects of a multilevel intervention, which may not be feasible in realworld settings. To overcome this issue and ensure the standard of rigorous scientific evaluation, we will use a stepped wedge cluster randomized trial design to evaluate the effectiveness and impact of our multilevel intervention. The stepped wedge is a pragmatic study design and retains some elements of randomization as it is a controlled trial [97-99]. The stepped wedge design includes an initial period of no exposure. Then one cluster (or group of clusters) will be randomized to cross from the control to the intervention at regular intervals. In the end, all clusters will be exposed to the intervention component but not at the same point in time. Since each cluster contributes to both exposed and unexposed observations and acts as its own control, such design can enhance the precision of the study [97]. Furthermore, the feature of multiple data collection points in stepped wedge design allows the researcher to capture the impact of an intervention when such impact develops over time, or when the intervention needs an initial period of adjustment before becoming embedded in the setting. This feature is especially valuable for the ACCSIS-Chicago study because we expect our outcomes will change over time rather than at a discrete-time point.

We will have four clusters of clinics from four different federally qualified health systems. Each cluster will have 5 to 12 clinics and a total of 20 to 35 primary care providers (internists, family practice physicians, nurse practitioners, and physician assistants). Our biostatistician will conduct the randomization independently from the research team. Figure 4 shows the stepped wedge study design. The study design will include an initial 3month period during which no intervention components will be implemented. We will use this 3-month waiting period to collect baseline outcome data from the previous year. After the 3-month waiting period, we will randomize two clusters to cross from the control to the intervention and the remaining clusters to follow 3 months later. All clusters will stay at the same phase for 9 months, followed by a 3-month transition period, and then cross over to the next phase. We will collect quarterly data throughout the 4-year study period. 
Table 2 Implementation strategies for each intervention component

\begin{tabular}{|c|c|}
\hline Intervention Component & Implementation Strategy \\
\hline Provider and staff education - academic detailing & $\begin{array}{l}\text { - Interactive: audience response system } \\
\text { - } \quad \text { Short session: } 15-20 \text { minutes. } \\
\text { - Individual and small group }\end{array}$ \\
\hline Provider reminder & $\begin{array}{ll}\text { - } & \text { Electronic medical record prompt } \\
\text { - } & \text { Chart flagging } \\
\text { - } & \text { Morning huddles }\end{array}$ \\
\hline Provider assessment and feedback & $\begin{array}{l}\text { - Quarterly report card } \\
\text { - Transparency: peer-to-peer and clinic-to-clinic } \\
\text { comparison } \\
\text { - Incentive } \\
\text { - Quality improvement initiative }\end{array}$ \\
\hline Patient reminder for FIT and Colonoscopy & $\begin{array}{l}\text { - } \quad \text { Provider and staff training } \\
\text { - Print materials for patients } \\
\text { - Personalize message with ordering provider's name } \\
\text { and clinic name }\end{array}$ \\
\hline Patient Navigation & $\begin{array}{l}\text { - } \quad \text { Centralization } \\
\text { - } \quad \text { Weekly meetings } \\
\text { - } \quad \text { Activity log }\end{array}$ \\
\hline
\end{tabular}

\section{Study sites}

We will partner with four FQHCs, two in Illinois and two in Indiana. Together, our FQHC partners have 40 primary care clinics and 130 primary care providers and served 162,000 individual patients in 2018. Of the 162,000 patients, $78 \%$ were racial minorities, $93 \%$ live at or below $200 \%$ poverty, and $17 \%$ were uninsured. The CRC screening rates among our partners range from 25 to $43 \%$.

\section{Data sources and outcome measures}

During the first 6 months, we will collect baseline data on primary outcomes (Table 3) from the EHR systems. After the initiation of the implementation process, we will collect primary outcome measures each quarter until the conclusion of the study completion.

For the patient navigation component, we will also collect data from the navigator activity logs, including the number of patients being served, length of the encounter, types of encounter (e.g., transportation arrangement or making the appointment), and types of action taken. Furthermore, we will also ask patients who received navigation services to complete a patient satisfaction survey adapted from the patient satisfaction survey developed by the NCI-sponsored Patient Navigation Research Program [100].

\section{Process evaluation}

We will assess the potential causal and contextual factors that may be associated with observed outcomes at the provider and clinic levels. After completion of each phase, we will send all providers at our partner clinics a survey to assess their exposure to and experience with the intervention component. Furthermore, we will evaluate the changes in organizational culture and climate that may ascertain potential mechanisms of impact using the 29-survey items we developed based on the CFIR.

\section{Data analysis plan}

We will conduct descriptive analyses, as well as within-group and between-group changes over time. Furthermore, to evaluate the four-component intervention effects, we will take a step-by-step approach. First, each intervention effect at each time phase will be estimated separately using linear-mixed effects models. The estimate of intervention effect in each model will be used to test if additional intervention component significantly affects outcome measures. Next, we will develop a grand model, 


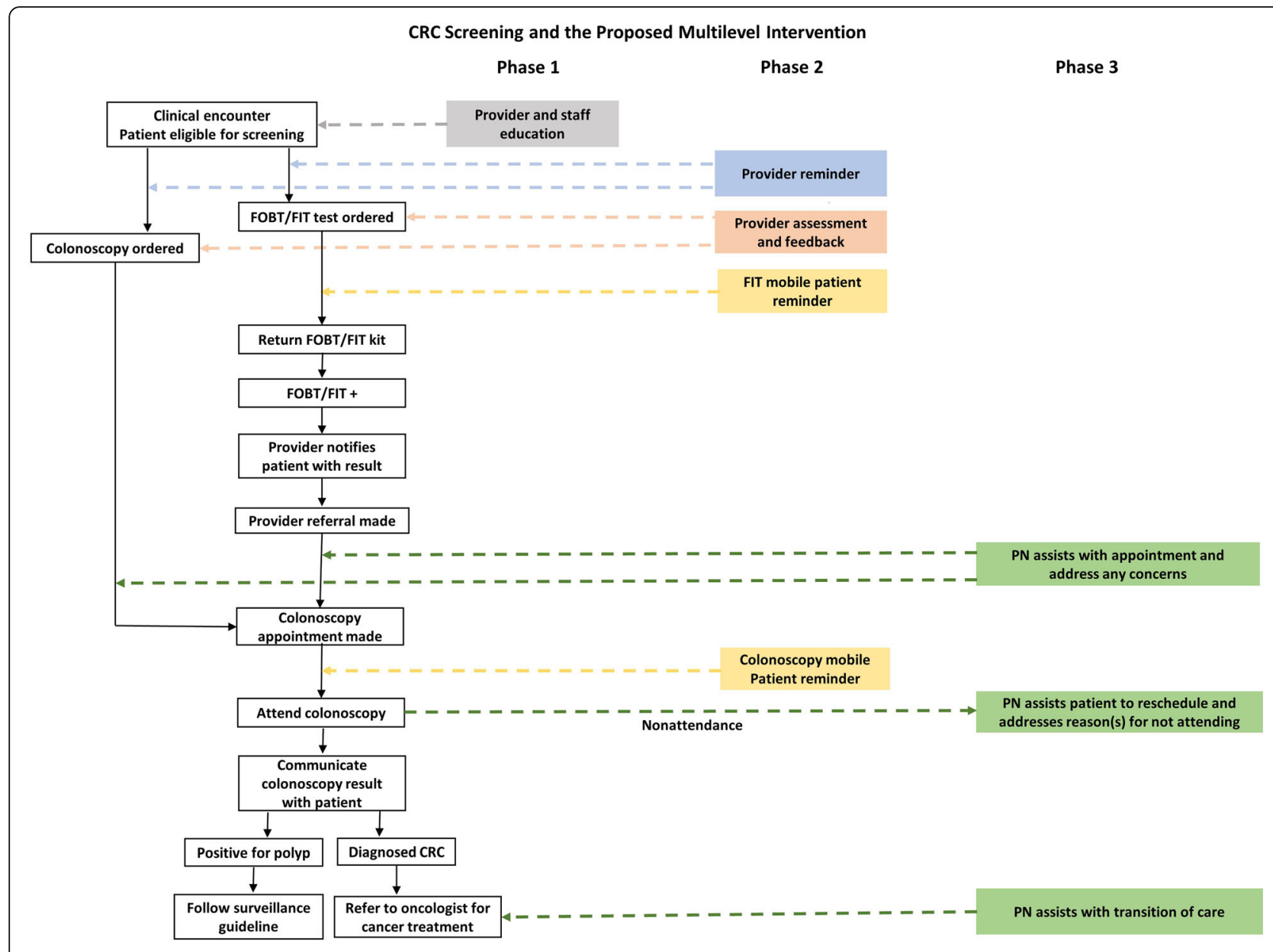

Fig. 3 CRC screening and the proposed multilevel intervention

including all intervention components simultaneously in addition to the models described above.

In this study, patients are nested within providers, and providers are nested within clinics. The problem with nested data structures is that they violate the independence assumption of traditional regression models. Thus, we will use multilevel modeling to analyze our data. We will start with the unconditional (null) three-level random intercept models:

Level $-1: Y_{i j k}=\beta_{0 j \mathrm{k}}+e_{\mathrm{ijk}}$

Level $-2: \beta_{0 j k}=\beta_{0 \mathrm{k}}+u_{\mathrm{jk}}$

Level - 3: $\beta_{\mathrm{Ok}}=\beta_{0}+\nu_{\mathrm{k}}$

where $Y_{i j k}$ is the observed outcome for patient $i$ with provider $j$ in clinic $k . \beta_{0}$ is the mean response across all

\begin{tabular}{|c|c|c|c|c|c|c|c|c|c|c|c|c|c|c|c|c|c|}
\hline \multirow{3}{*}{$\begin{array}{l}\text { Roll out } \\
\text { Group }\end{array}$} & \multicolumn{17}{|c|}{ Stepped Wedge Study Design } \\
\hline & & \multicolumn{4}{|c|}{ Year 1} & \multicolumn{4}{|c|}{ Year 2} & \multicolumn{4}{|c|}{ Year 3} & \multicolumn{4}{|c|}{ Year 4} \\
\hline & & Q1 & Q2 & Q3 & Q4 & Q1 & Q2 & Q3 & Q4 & Q1 & Q2 & Q3 & Q4 & Q1 & Q2 & Q3 & Q4 \\
\hline \multirow{2}{*}{ Group 1} & FQHC & $\frac{\pi}{\mathbb{5}}$ & \multicolumn{3}{|c|}{ Phase 1} & & \multicolumn{3}{|c|}{ Phase 2} & & \multicolumn{3}{|c|}{ Phase 3} & \multirow{2}{*}{\multicolumn{3}{|c|}{$\begin{array}{c}\text { Phase of } \\
\text { sustainability }\end{array}$}} & \\
\hline & Cluster 2 & $\stackrel{\mathscr{g}}{\underline{\underline{a}}}$ & \multicolumn{3}{|c|}{ Phase 1} & & & hase & & & \multicolumn{3}{|c|}{ Phase 3} & & & & \\
\hline \multirow{2}{*}{ Group 2} & Cluster 3 & 疍 & & \multicolumn{3}{|c|}{ Phase 1} & & \multicolumn{3}{|c|}{ Phase 2} & & \multicolumn{3}{|c|}{ Phase 3} & & & \\
\hline & Cluster 4 & 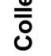 & & \multicolumn{3}{|c|}{ Phase 1} & & \multicolumn{3}{|c|}{ Phase 2} & & \multicolumn{3}{|c|}{ Phase 3} & & & \\
\hline
\end{tabular}

Fig. 4 Stepped wedge study design 
Table 3 Outcome measures for the multilevel intervention

\begin{tabular}{|c|c|c|c|c|}
\hline Outcome & Operational Definition & Data Sources & Data Collection Period & Level of Data Analysis \\
\hline Patient CRC screening rates & $\begin{array}{l}\text { Numerator: \# of patients completed CRC screening } \\
\text { Denominator: \# of patients eligible for CRC screening }\end{array}$ & $\begin{array}{l}\text { - EMR } \\
\text { - Chart reviews as needed }\end{array}$ & Every quarter & $\begin{array}{l}\text { Level 1, Level 2, Level } 3 \text {, } \\
\text { Level } 4\end{array}$ \\
\hline $\mathrm{CRC}$ ordering rates & $\begin{array}{l}\text { Numerator: \# of patients received order for CRC screening } \\
\text { Denominator: \# of patient eligible for CRC screening }\end{array}$ & $\begin{array}{l}\text { - EMR } \\
\text { - Chart reviews as needed }\end{array}$ & Every quarter & Level 2, Level 3, Level 4 \\
\hline $\begin{array}{l}\text { Patient notification rates } \\
(\text { FOBT/FIT +) }\end{array}$ & $\begin{array}{l}\text { Numerator: \# of patients notified } \\
\text { Denominator: \# of patients had FOBT/FIT + }\end{array}$ & $\begin{array}{l}\text { - } \quad \text { EMR } \\
\text { - Chart review as needed }\end{array}$ & Every quarter & Level 2, Level 3, Level 4 \\
\hline $\begin{array}{l}\text { Time to notification of } \\
\text { positive results }\end{array}$ & $\begin{array}{l}\text { \# of days between notifying a patient with a positive result and the result } \\
\text { available to the provider }\end{array}$ & $\begin{array}{l}\text { - EMR } \\
\text { - Chart reviews as needed }\end{array}$ & Every quarter & Level 2, Level 3, Level 4 \\
\hline $\begin{array}{l}\text { Time to referral for follow-up } \\
\text { diagnostic evaluation }\end{array}$ & \# of days between the referral and the result available to the provider & $\begin{array}{l}\text { - EMR } \\
\text { - Chart reviews as needed }\end{array}$ & Every quarter & Level 2, Level 3, Level 4 \\
\hline $\begin{array}{l}\text { Follow-up diagnostic } \\
\text { evaluation completion rates }\end{array}$ & $\begin{array}{l}\text { Numerator: \# of patients completed diagnostic evaluation as ordered } \\
\text { Denominator:\# of diagnostic evaluation referral made }\end{array}$ & $\begin{array}{l}\text { - EMR } \\
\text { - Chart reviews as needed }\end{array}$ & Every quarter & Level 2, Level 3, Level 4 \\
\hline $\begin{array}{l}\text { Time to completed } \\
\text { diagnostic evaluation }\end{array}$ & $\begin{array}{l}\text { \# of days between the positive FOBT/FIT result available to the provider } \\
\text { and the completion of the diagnostic evaluation }\end{array}$ & $\begin{array}{l}\text { - EMR } \\
\text { - Chart reviews as needed }\end{array}$ & Every quarter & Level 2, Level 3, Level 4 \\
\hline
\end{tabular}

clinics. " $v_{\mathrm{k}}$ " is the random effect of clinic $k$, " $u_{\mathrm{jk}}$ " is the random effect of provider $j$ in clinic $k$, and $\mathrm{e}_{\mathrm{ijk}}$ is the residual error. The random effects and residual errors are assumed to be normally distributed and independent of one another. Putting these submodels together yields the (null) 3-level model as: $Y_{i j k}=\beta_{0}+\nu_{\mathrm{k}}+u_{\mathrm{jk}}+e_{\mathrm{ijk}}$

Adding predictor variables to the models is straightforward. For example, the 3-level model with one predictor measured at each level is $Y_{i j k}=\beta_{0}+\beta_{1} X_{1 i j k}+\beta_{2} X_{2 j k}+$ $\beta \mathrm{X}_{3 \mathrm{k}}+v_{\mathrm{k}}+u_{\mathrm{jk}}+e_{\mathrm{ijk}}$, where $\beta_{0}+\beta_{1} \mathrm{X}_{1 \mathrm{ijk}}+\beta_{2} \mathrm{X}_{2 \mathrm{jk}}+\beta \mathrm{X}_{3 \mathrm{k}}$ is the fixed effect component of the model and $v_{\mathrm{k}}+u_{\mathrm{jk}}$ $+e_{\mathrm{ijk}}$ is the random effect component of the model. The fixed effect of the model specifies the overall mean relationship between the response and the predictor variables. The random effect component of the model specifies how the provider and clinic specific relationships differ from the overall mean relationship. To assess any cross-level interaction effects, we will add a product term to the model. For example, the model for a level-1 predictor (e.g., age) interacting with a level-2 predictor: $Y_{i j \mathrm{k}}=\beta_{0}+\beta_{1} \mathrm{X}_{1 \mathrm{ijk}}+\beta_{2} \mathrm{X}_{2 \mathrm{jk}}+\beta_{3}\left(\mathrm{X}_{1 \mathrm{ijk}} \bullet \mathrm{X}_{2 \mathrm{jk}}\right)+v_{\mathrm{k}}+u_{\mathrm{jk}}$ $+e_{\mathrm{ijk}}$. Since one of the patient-level (level-1) outcome measures is dichotomous (whether the patient adherence to follow-up diagnostic evaluation after a positive result within 9 months or not), we will use a multilevel logistic model for binary data. In this case, the level-1 model is in terms of the logit of the response of the level-1 binary outcome $Y_{i j k}$, for example:

Level $-1: \log \left[P_{\mathrm{ijk} /}\left(1-P_{\mathrm{ijk}}\right)\right]=\beta_{0 \mathrm{jk}}+\beta_{1} \mathrm{X}_{\mathrm{ijk}}$.

In this study, we will use the restricted maximum likelihood (REML) to estimate variances. REML treats the regression coefficients as unknown quantities to be an estimate based on sample data and subtracts the needed degree of freedom when computing variance estimates. Since REML only allows for tests of models that differ in their variances, we will calculate the intraclass correlation (ICC) to access the variation in response variable across providers and clinics before testing the models.
Statistical analyses will be conducted using Statistical Package for the Social Science (SPSS) version 26.0 and Stata version 15, with the significance level set at alpha $\leq 0.05$.

\section{Sample size and power}

Although we will implement the multilevel intervention over three phases, the power of the study is calculated based on the phase 1 period only. In our pilot study, the baseline rate in the intervention group was $30.8 \%$, and it went up to $40.7 \%$ in the year 2019 . We observed the intra-class correlation as 0.135 . Considering four components of the intervention, we lower the significance level to $0.05 / 4=0.017$. We will recruit a total of 40 clinics and 130 providers across four partner healthcare systems (clusters) (see Table 3). Although the number of clinics and providers varies between clusters, we calculate the power based on the average number of providers, which is 32 providers per cluster. Under these conditions, the study will have power in excess of $90 \%$ to detect an increase in the CRC screening rate of $32 \%$. Table 4 shows the levels of powers for different sample sizes and effect sizes for reference.

\section{Discussion}

\section{Limitations and related considerations \\ Using EHR data for research}

The adoption of electronic health records (EHRs) has significantly increased the amount of detailed patient health information available today, which would be difficult to obtain using survey data alone. Using data collected in EHRs can improve clinical research efficiency, including the investigation of relationships between interventions and outcomes [101, 102]. However, raw EHR data are disorganized and full of uncodified variables. There remain immense hurdles to extract and apply EHR data effectively for research purposes. Furthermore, our partners use different EHR systems, including Epic, 
Table 4 Powers with respect to different effective sizes

\section{Powers With Respect to Different Effective Sizes}

\begin{tabular}{|c|c|c|c|}
\hline \multirow{2}{*}{$\begin{array}{c}\text { Sample size } \\
\text { (Number of provider per cluster) }\end{array}$} & \multicolumn{3}{|c|}{$\begin{array}{l}\text { CRC Screening rate after the intervention } \\
\text { (Baseline rate is } 30.8 \%)\end{array}$} \\
\hline & $32 \%$ & $35 \%$ & $40 \%$ \\
\hline 20 & $76.3 \%$ & $84.6 \%$ & $93.6 \%$ \\
\hline 25 & $86.3 \%$ & $92.4 \%$ & $97.7 \%$ \\
\hline 30 & $92.4 \%$ & $96.4 \%$ & $99.2 \%$ \\
\hline
\end{tabular}

NextGen, and Athena. We have learned valuable techniques and steps to capture data from different EHR systems in our previous studies. We will work with our health system partners to develop a plan for data capturing and train a data coordinator at each site to effectively extract the required data and locate other data sources that may fill the gaps. Furthermore, we will implement a data validation process by randomly selecting $5 \%$ of the medical records and manually reviewed to ensure the integrity and quality of the data.

\section{Evaluating the impact on follow-up diagnostic colonoscopy}

Only about $4 \%$ to $6 \%$ of patients who use fecal occult blood test (FOBT) or fecal immunochemical test (FIT) as a screening modality will have a positive result and need follow-up diagnostic evaluation. The success of this study to measure improvement in follow-up rates will depend on the screening rates using FIT/FOBT among our partner FQHCs. To address this potential pitfall, we will continue to monitor the CRC screening rates among our partner FQHCs and the use of FIT/FOBT as a screening modality.

\section{Potential for impact and implications and plan for dissemination}

There is a pressing need to reduce disparities in CRC outcomes, especially among racial/ethnic minority populations and among populations who live in poverty. There is also a need to understand the design and implementation of pragmatic studies in real-world settings within healthcare systems. Single-level interventions are often insufficient to lead to sustainable changes. Multilevel interventions, those that target two or more levels of changes, are needed to address multilevel influences simultaneously. Multilevel interventions with multiple components will affect not only the desired outcomes but also each other. How to take advantage of multilevel interventions and how to implement such interventions and evaluate their effectiveness are the ultimate goals of this study. ACCSIS-Chicago will provide the needed evidence for future multilevel interventional studies, especially in increasing CRC screening, follow-up, and referral-to-care. Furthermore, it will demonstrate best practice for how multilevel interventions can be scaledup nationwide to reduce the burden of CRC among racial/ethnic and low-income populations that usually seek health care in resource strained safety-net systems.

The rigor, design, feasibility, and high likelihood of success of this study will provide crucial evidence regarding multilevel interventions that target three levels of influences (organization, provider, and individual) to improve CRC screening, follow-up, and referral-to-care. We will disseminate these findings through peerreviewed publications, presentations, and professional meetings. Throughout this effort, we will share data following the National Cancer Institute NCI) policies and submit standard data elements to the NCI data repository.

\section{Abbreviations \\ CRC: Colorectal cancer; SES: Low social-economic status; FQHC: Federally qualified health center; FOBT: Fecal occult blood test; FIT: Fecal \\ immunochemical test; TPB: Theory of Planned Behavior Model; ACCSIS- Chicago: Accelerating Colorectal Cancer Screening and Follow-Up Through Implementation Science - Chicago; CDC: Center for Disease Control and Prevention; EHR: Electronic health record; SMS: Short message service; ORA: Organization readiness assessment; CFIR: Consolidated Framework for Implementation Research}

\section{Acknowledgements}

We would like to thank our federally qualified health center partners who volunteer for participation. We are deeply grateful to the ACCSIS Coordinate Center, RTI International, and other ACCSIS grantees who have provided invaluable feedback on this study.

\section{Authors' contributions}

$\mathrm{KK}, \mathrm{BP}, \mathrm{DH}$, and $\mathrm{HL}$ conceived the study design. $\mathrm{KK}, \mathrm{BP}, \mathrm{DH}, \mathrm{DL}, \mathrm{FR}, \mathrm{MJ}, \mathrm{MQ}$, $S M L$, and $H L$ developed the study procedures and protocol. KK, BP, FR, MJ, and $\mathrm{HL}$ facilitated partnerships with federally qualified health centers. KK and $\mathrm{HL}$ drafted the manuscript. All authors have read, edited, and approved the final manuscript.

\section{Funding}

This work is supported through the National Cancer Institute Moonshot Initiative (5UH3CA233229-03).

Availability of data and materials Not applicable. 


\section{Ethics approval and consent to participate}

The protocol for the study has been reviewed and approved by the University of Chicago Institutional Review Board (IRB19-1496).

\section{Consent for publication}

Not applicable.

\section{Competing interests}

The authors declare that they have no competing interests.

\section{Author details}

'Center for Asian Health Equity, University of Chicago, 5841 S. Maryland Ave, MC1140, Chicago, IL 60637, USA. ${ }^{2}$ University of Chicago Medicine Hematology and Oncology, 5841 S Maryland Ave, Chicago, IL 60637, USA. ${ }^{3}$ Department of Public Health Sciences, University of Chicago Biological Sciences, 5841 S. Maryland Ave, Rm W-254, MC2000, Chicago, IL 60637, USA. ${ }^{4}$ Division of General Internal Medicine \& Geriatrics, Feinberg School of Medicine, Northwestern University, 750 N. Lake Shore Dr., 10th Floor, Chicago, IL 60611, USA. ${ }^{5}$ Department of Internal Medicine, Section of General Internal Medicine, University of Chicago, 5841 S Maryland Ave, Chicago, IL 60637, USA.

\section{Received: 1 September 2020 Accepted: 10 September 2020} Published online: 29 October 2020

\section{References}

1. Siegel RL, Miller KD, Jemal A. Cancer statistics, 2019. CA Cancer J Clin. 2019; 69:7-34.

2. Joseph DA, King JB, Dowling NE, Thomas CC, Richardson LC. Vital sign: colorectal cancer screening test use-United States, 2018. MMWR. 2020; 69(10):253-9.

3. CDC. Behavioral Risk Factor Surveillance System Survey data. Atlanta, Georgia: U.S. Department of Health and Human Services; 2010.

4. Cyhaniuk A, Coombes ME. Longitudinal adherence to colorectal cancer screening guidelines. Am J Manag Care. 2016;22:105-11.

5. Etzioni DA, Ponce NA, Babey SH, et al. A population-based study of colorectal cancer test use: results from the 2001 California Health Interview Survey. Cancer. 2004;101:2523-32.

6. List DT, Baker DW. Understanding current racial/ethnic disparities in colorectal cancer screening in the United States: the contribution of socioeconomic status and access to care. Am J Prev Med. 2014;46:228-36.

7. Klabunde CN, Cronin KA, Breen N, Waldron WR, Ambs AH, Nadel MR. Trends in colorectal cancer test use among vulnerable populations in the United States. Cancer Epidemiol Biomark Prev. 2011;20:1611-21.

8. Gupta S, Tong L, Allison JE, et al. Screening for colorectal cancer in a safetynet health care system: access to care is critical and has implications for screening policy. Cancer Epidemiol Biomark Prev. 2009;18:2373-9.

9. 2018 Program grantee comparison data. Health Resources and Service Administration. 2018. https://bphc.hrsa.gov/uds/datacenter.aspx. Accessed 18 Apr 2020.

10. Daly JM, Levy BT, Moss CA, Bay CP. System strategies for colorectal cancer screening at Federally Qualified Health Centers. Am J Public Health. 2015; 105(1):212-8.

11. Inadomi JM, Vijan S, Janz NK, et al. Adherence to colorectal cancer screening: a randomized clinical trial of competing strategies. Arch Intern Med. 2012;172:575-82.

12. Singal AG, Gupta S, Tiro JA, et al. Outreach invitations for FIT and colonoscopy improve colorectal cancer screening rates: a randomized controlled trial in a safety-net health system. Cancer. 2016:122:456-63.

13. Hawley ST, Volk RJ, Krishnamurthy P, Jibaja-Weiss M, Vernon SW, Kneuper S. Preferences for colorectal cancer screening among racial/ethnically diverse primary care patients. Med Care. 2008;46:S10-6.

14. Callen JL, Westbrook Jl, Georgiou A, et al. failure to follow-up test results for ambulatory patients: a systematic review. J Gen Intern Med. 2012;27(10): 1334-48.

15. Myers RE, Balshem AM, Wolf TA, et al. Screening for colorectal neoplasia: physicians' adherence to complete diagnostic evaluation. Am J Public Health. 1993:83(11):1620-2.

16. Chauvin P, Josselin JM, Heresbach D. The influence of waiting times on cost-effectiveness: a case study of colorectal cancer mass screening. Eur J Health Econ. 2014;15(8):801-12.
17. McCarthy AM, Kim JJ, Beaber EF, et al. Follow-up of abnormal breast and colorectal cancer screening by race/ethnicity. Am J Prev Med. 2016;51(4): $507-12$.

18. Tosteson ANA, Beaber EF, Tiro J, et al. Variation in screening abnormality rates and follow-up of breast, cervical and colorectal cancer screening within the PROSPR consortium. J Gen Intern Med. 2015;31(4):372-9.

19. Carlson CM, Kirby KA, Casadei MA, Partin MR, Kistler CE, Walter LC. Lack of follow-up after fecal occult blood testing in older adults: inappropriate screening or failure to follow-up? Arch Intern Med. 2011;171:249-56.

20. Choi KS, Lee HY, Jun JK, Shin A, Park EC. Adherence to follow-up after a positive fecal occult blood test in an organized colorectal cancer screening program in Korea, 2004-2008. J Gastroenterlo Hepatol. 2012; 27:1070-7.

21. Ferrat E, le Breton J, Veerabudun K, Bercier S, Brixi Z, Khoshnood B, et al. Colorectal cancer screening: factors associated with colonoscopy after a positive fecal occult blood test. Br J Cancer. 2013;109:1437-44.

22. Miglioretti DL, Rutter CM, Bradford SC, Zauber AC, Kessler LG, Feuer EJ, et al. Improvement in the diagnostic evaluation of a positive fecal occult blood test in an integrated health care organization. Med Care. 2008;46:S91-106.

23. Rao SK, Schilling TF, Sequist TD. Challenges in the management of positive fecal occult blood tests. J Gen Intern Med. 2009;24:356-60.

24. Shields HM, Weiner MD, Henry DR, Lloyd JA, Ransil BJ, Lamphier DA, et al. Factors that influence the decision to do an adequate evaluation of a patient with a positive stool for occult blood. Am J Gastroenterol. 2001;96: 196-203.

25. Fisher DA, Jeffreys A, Coffman CJ, Fasanella K. Barriers to full colon evaluation for a positive fecal occult blood test. Cancer Epidemiol Biomark Prev. 2006;15:1232-5.

26. Morris S, Baio G, Kendall E, von Wagner C, Wardle J, Atkin W, et al. Socioeconomic variation in uptake of colonoscopy following a positive fecal occult blood test result: a retrospective analysis of the NHS Bowel Screening Programme. Br J Cancer. 2012;107:765-71.

27. Pasat L, Rabeneck L, Kiefer L, Mai V, Ritvo P, Sullivan T. Endoscopic follow-up of positive fecal occult blood testing in the Ontario FOBT Project. Can J Gastroenterol. 2007;21:379-82.

28. Issaka RB, Singh MH, Oshima SM, Laleau VJ, Rachocki CD, Chen EH, et al. Inadequate utilization of diagnostic colonoscopy following abnormal FIT results in an integrated safety-net system. Am J Gastroenterol. 2017;112:375-82.

29. Chubak J, Garcia MP, Burnett-Hartman AN, Zheng Y, Corley DA, Halm EA, et al. Time to colonoscopy after positive fecal blood test in four U.S. health care systems. Cancer Epidemiol Biomark Prev. 2016;25(2):344-50.

30. Oluloro A, Petrik AF, Turner A, Kapka T, Rivelli J, Carney PA, et al. Timeliness of colonoscopy after abnormal fecal test results in a safety net practice. J Community Health. 2016;41:864-70.

31. Engel GL. The clinical application of the biopsychosocial approach. I. In: Frankel RM, Quill TE, McDaniel S, editors. The Biopsychosocial Approach: Past, Present, Future. NY: University of Rochester Press; 2003. p. 1-20.

32. Sallis JF, Cervero RB, Ascher W, Henderson KA, Kraft MK, Kerr J. An ecological approach to creating active living communities. Annu Rev Public Health. 2006;27:297-322.

33. Centers for Disease Control and Prevention. Colorectal Cancer Control Program (CRCCP): Social Ecological Model: CDC; 2015. https://www.cdc.gov/ cancer/crccp/sem.htm. Access 17 Apr 2017.

34. Clauser SB, Taplin SH, Foster MK, Fagan P, Kaluzny AD. Multilevel intervention research: lessons learned and pathways forward. J Natl Cancer Inst Monogr. 2012;2012(44):127-33.

35. Yano EM, Green LW, Glanz K, Ayanian JZ, Mittman BS, Chollette V, et al. Implementation and spread of interventions into the multilevel context of routine practice and policy: implications for the cancer care continuum. J Natl Cancer Inst Monogr. 2012;2012(44):86-99.

36. Flood AB, Fennell ML, Devers KJ. Health reforms as examples of multilevel interventions in cancer care. J Natl Cancer Inst Monogr. 2012;2012(44):80-5.

37. Taplin SH, Anhang Price R, Edwards HM, Foster MK, Breslau ES, Chollette V, et al. Introduction: understanding and influencing multilevel factors across the cancer care continuum. J Natl Cancer Inst Monogr. 2012;2012(44):2-10.

38. Stange KC, Breslau ES, Dietrich AJ, Glasgow RE. State-of-the-art and future directions in multilevel interventions across the cancer control continuum. J Natl Cancer Inst Monogr. 2012;2012(44):20-31.

39. Edwards HM, Taplin SH, Chollette V, Clauser SB, Das IP, Kaluzny AD. Summary of the multilevel interventions in health care conference. J Natl Cancer Inst Monogr. 2012;2012(44):123-6. 
40. Klabunde CN, Vernon SW, Nadel MR, Breen N, Seeff LC, Brown ML. Barriers to colorectal cancer screening; a comparison of reports from primary care physicians and average risk adults. Med Care. 2005;43:939-44.

41. Wee CC, Mccarthy EP, Phillips RS. Factors associated with colon cancer screening: the role of patient factors and physician counseling. Prev Med. 2005:41:23-9.

42. Brenes GA, Paskett ED. Predictors of adoption for colorectal cancer screening. Prev Med. 2000;31:410-6.

43. Zapka JM, Klabunde CN, Arora NK, Yuan G, Smith JL, Kobrin SC. Physicians' colorectal cancer screening discussion and recommendation patterns. Cancer Epidemiol Biomark Prev. 2001;20:509-21.

44. Teng EJ, Friedman LC, Green CE. Determinants of colorectal cancer screening behavior among Chinese Americans. Psycho-Oncology. 2006;15: 374-81

45. DeVoe JE, Fryer GE, Phillips R, Green L. Receipt of preventive care among adults: insurance status and usual source of care. Am J Public Health. 2003, 93:786-91.

46. Ioannou GN, Chapko MK, Dominitz JA. Predictors of colorectal cancer screening participation in the United States. Am J Gastroenterol. 2003;98: 2082-91.

47. Palmer RC, Vernon SW, Nadel MR, et al. Social disparities across the continuum of colorectal cancer: a systematic review. Cancer Causes Control. 2005;16:55-61.

48. Carcaise-Edinboro P, Bradley CJ. Influence of patient-provider communication on colorectal cancer screening. Med Care. 2008;46:738-45.

49. Wackerbarth SB, Tarasenko YN, Joyce JM, Haist SA. Physician colorectal cancer screening recommendations: an examination based on informed decision making. Patient Educ Couns. 2007;66:43-50.

50. Farmer MM, Bastani R, Kwan I, Belman M, Ganz PA. Predictors of colorectal cancer screening from patients enrolled in a managed care health plan. Cancer. 2008;112:30-8

51. Gilbert A, Kanerak N. Colorectal cancer screening: physician recommendation is influential advice to Marylanders. Prev Med. 2005;41: 367-79.

52. Mandleson MT, Curry SJ, Anderson IA, Nadel MR, et al. Colorectal cancer screening participation by older women. Am J Prev Med. 2000;19:149-54.

53. Jo AM, Maxwell AE, Rick AJ, Cha J, Bastani R. Why are Korean American physicians reluctant to recommend colorectal cancer screening to Korean American patients? J Immigr Minor Health. 2009;11:302-9.

54. Fritz C, Naylor K, Kim K. Knowledge of polyp history and recommended follow-up among a predominately African American patient population and the impact of patient navigation. J Racial Ethn Health Disparities. 2016;3: 403-12.

55. Laiyem AO, Adebogun AO, Doubnia CA, et al. Influence of provider discussion and specific recommendation on colorectal cancer screening uptake among U.S. adults. Prev Med. 2014;67:1-5.

56. Peterson EB, Ostroff JS, DuHamel KN, D'Agostino TA, Hernandez M, Canzona MR, Bylund CL. Impact of provider-patient communication on screening adherence: a systematic review. Prev Med. 2016:93:96-105.

57. Stewart M, Brown JB, Donner A, et al. The impact of patient-centered care on outcomes. J Fam Pract. 2000;49:796-804

58. Golin C, DiMatteo MR, Duan N, Leake B, Gelberg L. Impoverished diabetic patients whose doctors facilitate their participation in medica decision making are more satisfied with their care. J Gen Intern Med. 2002:17:857-66

59. Gattellari M, Butow PN, Tattersall MH. Sharing decisions in cancer care. Soc Sci Med. 2001;52:1865-78.

60. Kaplan SH, Greenfield S, Gandek B, Rogers WH, Ware JE. Characteristics of physicians with participatory decision-making styles. Ann Intern Med. 1996; 124:497-504.

61. Ling BS, Schoen RE, Trauth JM, et al. Physicians encouraging colorectal screening: a randomized controlled trial of enhanced office and patient management on compliance with colorectal cancer screening. Arch Intern Med. 2009:169:47-55.

62. Roetzheim RG, Christman LK, Jacobsen PB, et al. A randomized controlled trial to increase cancer screening among attendees of community health centers. Ann Fam Med. 2004;2:294-300.

63. Ornstein S, Nemeth LS, Jenkins RG, Nietert PJ. Colorectal cancer screening in primary care: translating research into practice. Med Care. 2010;48:900-6.

64. Curry WJ, Lengerich EJ, Kluhsman BC, Graybill MA, Liao JZ, Schaefer EW, Spleen AM, Dignan MB. Academic detailing to increase colorectal cancer screening by primary care practices in Appalachian Pennsylvania. BMC Health Serv Res. 2011;11:112.

65. Dignan M, Shelton B, Slone SA, Tolle C, Mohammad S, Schoenberg, Pearce K, Van Meter Z, Ely G. Effectiveness of a primary care practice intervention for increasing colorectal cancer screening in Appalachian Kentucky. Prev Med. 2014;58:70-4.

66. Shankaran V, Luu TH, Nonzee N, Richey E, McKoy JM, Graffzivin J, et al. Costs and cost-effectiveness of a health care provider-directed intervention to promote colorectal cancer screening. J Clin Oncol. 2009; 27:5370-5.

67. Mader EM, Fox CH, Epling JW, Noronha GJ, Swanger CM, Wisiniewaki AM, Vitale K, Norton AL, Morley CP. A practice facilitation and academic detailing intervention can improve cancer screening rates in primary care safety net clinics. J Am Board Fam Med. 2016;29:533-42.

68. Baron RC, Melillo S, Rimer BK, et al. Intervention to increase recommendation and delivery of screening for breast, cervical, and colorectal cancers by healthcare providers: a systematic review of provider reminders. Am J Prev Med. 2010;38:110-7.

69. Uy C, Lopez J, Trinh-Shevrin C, Kwon SC, Sherman SE, Liang PS. Text messaging intervention on cancer screening rates: a systematic review. Med Internet Res. 2017:19:e296.

70. Wu Y, Liang Y, Zhou Q, Liu H, Lin G, Cai W, Li Y, Gi J. Effectiveness of short message service intervention to motivate people with positive results in preliminary colorectal cancer screening to undergo colonoscopy: a randomized controlled trial. Cancer. 2019;125:2252-61.

71. Azulay R, Valinsky L, Hershkowitz F, Maghezi R. Repeated automated mobile text messaging reminders for follow-up of positive fecal occult blood tests: randomized controlled trial. JMIR MHealth UHealth. 2019;7:e11114.

72. Walter B, Klare P, Aschenbeck J, Ludwig L, Dimopoulos N, Mayr M, Neu B, et al. Improving the quality and acceptance of colonoscopy with short message service: results from a randomized multicenter study (PERICLES II). Gastrointest Endosc. 2019:89:506-13.

73. Walter BM, Klare P, Neu B, Schmid RM, von DS. Development and testing of an automated 4-day text messaging guidance as an aid for improving colonoscopy preparation. JMIR Mhealth Uhealth. 2016;4:e75.

74. Lee YJ, Kim ES, Choi JH, Park KS, Cho KB, Jang BK, Chung WJ, Hwang JS. Impact of reinforced education by telephone and short message service on the quality of bowel preparation: a randomized controlled study. Endoscopy. 2015;47:1018-27.

75. Chen LA, Santos S, Jandorf L, Christie J, Castillo A, Winkel G, et al. A program to enhance completion of screening colonoscopy among urban minorities. Clin Gastroenterol Hepatol. 2008;6:443-50.

76. Christie J, Itzkowitz S, Lihau-Nkanza I, Castillo A, Redd W, Jandorf L. A randomized controlled trial using patient navigation to increase colonoscopy screening among low-income minorities. J Natl Med Assoc. 2008;100:278-84.

77. Jandorf L, Gutierrez Y, Christie J, Itzkowitz SH. Use of a patient navigator to increase colorectal cancer screening in an urban neighborhood health clinic. J Urban Health. 2005:82:216-24.

78. Lasser KE, Murillo J, Medlin E, Lisboa S, Valley-Shah L, Fletcher RH, et al. A multilevel intervention to promote colorectal cancer screening among community health center patients: results of a pilot study. BMC Fam Pract. 2009:10:37.

79. Percac-Lima S, Grant RW, Green A, Ashburner J, Gamba G, et al. A patient-tailored navigator program for colorectal cancer screening in a community health center: a randomized controlled trial. J Gen Intern Med. 2008;23:237-8

80. Myers RE, Bittner-Fagan H, Daskalakis C, et al. A randomized controlled trial of a tailored navigation and a standard intervention in colorectal cancer screening. Cancer Epidemiol Biomark Prev. 2013;22:109-17.

81. Green BB, Wang CY, Anderson Ml, et al. Automated intervention with stepped increases in support to increase uptake of colorectal cancer screening: a randomized trial. Ann Intern Med. 2013;15:301-11.

82. Percac-Lima S, Ashburner JM, Zai AH, et al. Patient navigation for comprehensive cancer screening in high-risk patients using a populationbased health information technology system: a randomized clinical trial. JAMA Intern Med. 2016;176:930-7.

83. Thamarasseril S, Phuket T, Chan C, Liu B, Wong RJ. The need for an integrated patient navigation pathway to improve access to colonoscopy after positive fecal immunochemical testing: a safety-net hospital experience. J Community Health. 2018;63:242-7. 
84. Freund KM, Battaglia TA, Calhoun E, Darnell JS, Dudley DJ, Fiscella K, et al, Impact of patient navigation on timely cancer care: the patient navigation research program. J Natl Cancer Inst. 2014;106:dju115.

85. Paskett ED, Katz ML, Post DM, Pennell ML, Young GS, Seiber EE, et al. The Ohio Patient Navigator Research Program: does the American Cancer Society Patient Navigation Model improve time to resolution in patients with abnormal screening tests? Cancer Epidemiol Biomark Prev. 2012;21(10): $1620-8$.

86. Green BB, Anderson ML, Wang CY, Vernon SW, Chubak J, Meenan RT, et al. Results of nurse navigator follow-up after a positive colorectal cancer screening test: a randomized trial. J Am Board Fam Med. 2014;27(6):789-95.

87. Wells KJ, Lee JH, Calcano ER, Meade CD, Rivera M, Fulp WJ, et al. A cluster randomized trial evaluating the efficacy of patient navigation in improving quality of diagnostic care for patients with breast or colorectal cancer abnormalities. Cancer Epidemiol Biomark Prev. 2012;21(10):1664-72.

88. DeGroff A, Gressard L, Glover-Kudon R, Rice K, Tharpe FS, Escoffery C, Gersten J, Butterly L. Assessing the implementation of a patient navigation intervention for colonoscopy screening. BMC Health Serv Res. 2019;19:803.

89. Rice K, Sharma K, Chunyu L, Gersten J, Butterly L, DeGroff A. Costeffectiveness of a patient navigation intervention to increase colonoscopy screening among low-income adults in New Hampshire. Cancer. 2019;125: $601-9$.

90. Rice K, Gressard L, DeGroff A, Gersten J, Robie J, Leadbetter S, Glover-Kudon $R$, Butterly L. Increasing colonoscopy screening in disparate populations: results from an evaluation of patient navigation in the New Hampshire Colorectal Cancer Screening Program. Cancer. 2017;123:3356-66.

91. Damschroder L, Aron DC, Keith RE, Kirsh SR, Alexander JA, Lowery JC. Fostering implementation of health services research findings into practice: a consolidated framework for advancing implementation science. Implement Sci. 2009;4:50.

92. Kegler MC, Liang S, Weiner BJ, Tu SP, Friedman DB, Glenn BA, et al. Measuring constructs of the Consolidated Framework for Implementation Research in the context of increasing colorectal cancer screening in federally qualified health center. Health Serv Res. 2018;53:4178-203.

93. Walker TJ, Rodriguez SA, Vernon SW, Savas LS, Frost EL, Fernandez ME, Validity and reliability of measures to assess constructs from the inner setting domain of the Consolidated Framework for Implementation Research in a pediatric clinic network implementing HPV programs. BMC Health Serv Res. 2019;19:205.

94. Fernandez ME, Walker TJ, Weiner BJ, Calo WA, Liang S, Risendal B, et al. Developing measures to assess constructs from the inner setting domain of the Consolidated Framework for Implementation Research. Implement Sci. 2018;13:52.

95. Helfrich CD, Li YF, Sharp ND, Sales AE. Organizational readiness to change assessment (ORCA): developing of an instrument based on the Promoting Action on Research in Health Services (PARHS) framework. Implement Sci. 2009:4:38.

96. Cleary PD, Gross CP, Zaslavsky AM, Taplin SH. Multilevel interventions: study design and analysis issues. J Natl Cancer Inst Monogr. 2012;44:49-55.

97. Hemming K, Haines TP, Clinton PJ, Girling AJ, Lilford RJ. The stepped wedge cluster randomized trial: rationale, design, analysis, and reporting. BMJ. 2015; 351:h391. https://doi.org/10.1136/bmj.h391.

98. Hardly MA, Schillinger D, Shiboski S. Quasi-experimental designs in practicebased research setting: design and implementation considerations. J Am Board Fam Med. 2011;24:589-96.

99. Rhoda DA, Murray DM, Andridge RR, Pennell ML, Hade EM. Studies with staggered starts: multiple baseline designs and group-randomized trials. Am J Public Health. 2011;101:2164-9.

100. Carle AC, Jean-Pierre P, Winters P, Valverde P, Wells K, Simon M, Raich P, Patierno S, Katz M, Freund KM, Dudley D, Fiscella K. Psychometric evaluation of the patient satisfaction with logistical aspects of navigation (PSN-L) scale using item response theory. Med Care. 2014 Apr;52(4):354-61.

101. Dean BB, Lam J, Natoli JL, et al. Use of electronic medical records for health outcomes research: a literature review. Med Care Res Rev. 2009:66:611-38.

102. Tannen RL, Weiner MG, Marcus SM. Simulation of the Syst-Eur randomized control trial using a primary care electronic medical record was feasible. J Clin Epidemiol. 2006;59:254-64

\section{Publisher's Note}

Springer Nature remains neutral with regard to jurisdictional claims in published maps and institutional affiliations.

\section{Ready to submit your research? Choose BMC and benefit from:}

- fast, convenient online submission

- thorough peer review by experienced researchers in your field

- rapid publication on acceptance

- support for research data, including large and complex data types

- gold Open Access which fosters wider collaboration and increased citations

- maximum visibility for your research: over $100 \mathrm{M}$ website views per year

At BMC, research is always in progress.

Learn more biomedcentral.com/submissions 